\title{
Aggregate interference from WLAN in the TV white space by using terrain-based channel model
}

\author{
Konstantinos Koufos, Kalle Ruttik and Riku Jäntti \\ Communications and Networking Department \\ Aalto University, School of Electrical Engineering \\ Espoo, Finland \\ Konstantinos.Koufos@aalto.fi, Kalle.Ruttik@aalto.fi, Riku.Jantti@aalto.fi
}

\begin{abstract}
In this paper we model the TV white space usage by a WLAN type secondary network. We derive a model that incorporates spatially non-uniform distributed user demand and terrain-based radio channel model. We follow the approach proposed by Electronic Communications Committee (ECC) and describe the interference from the pixels in the secondary area to the pixels in the TV coverage area. The demand is described by the household density and the radio channel is evaluated as attenuation between the pixels. We also study how well a Poisson point process (PPP) based interference model describes the actual interference. We modify the PPP model so that it can describe non-uniform user distribution and terrain-based channel model. The proposed model describes the actual interference as a sum from multiple areas. We study how the size of the selected areas impacts the approximation of the actual interference distribution.
\end{abstract}

\section{INTRODUCTION}

The use of TV white space (TVWS) is limited by the secondary users generated aggregate interference. The aggregate interference depends on multiple system and environment parameters. The versatility of those parameters makes it difficult to capture them by one simple model. Because of that the initial TVWS assessments are based on many simplifications. The widely utilized models usually do not contain terrainbased attenuation information, user demand densities, etc [1].

The most prominent aggregate interference model assumes transmitters to be located as a Poisson point process (PPP) [2]. In its general form, the PPP incorporates a simple power law based path loss model and independent transmitters' transmission patterns. Such simplifications give a good initial approximation of the TVWS environment. They allow to investigate the general interference behavior and draw coarse conclusions about the available capacity. Nevertheless, the actual TVWS assessment and the derivation of the TV receivers protection criteria should be based on a more detailed and realistic description of the radio environment.

In this paper we adjust the PPP model with terrain-based channel model and nonuniform distribution of users. We use a pixel-based discretization of the environment. The pixel size is adopted from the interference estimation approach proposed by ECC [3]. In this paper we compute the attenuation between the pixels by using the Longley-Rice channel model with terrain data [4].

The proposed model is intended to modify the PPP model for realistic interference environment. The nonuniform user density has been incorporated into the PPP model in [5]. While the results in [5] describe the general interference change in case of nonuniform demand, the proposed model is not suitable to incorporate the known locations of users. In this paper we use the PPP model with the household density map. We split the secondary system service area into smaller areas where the user density inside each area is assumed to be constant. We illustrate how the size of the area impacts the estimated interference level. Also, we compare the results with the naive PPP model where the secondary users are uniformly distributed and all the users have the same channel attenuation model.

The proposed model is suitable for estimating the generated interference from randomly located transmitters. In contrast to the cellular secondary spectrum access where the locations of the base stations are arbitrary, the considered scenario resembles a WLAN type of secondary deployment. Also, unlike the ECC rules where the allocated power is location dependent, we consider low-power transmitters with fixed transmission power level. The target is to estimate the impact of secondary transmissions under a massive use of spectrum.

It is well known that the PPP model gives only a pessimistic estimation of the aggregate interference from a WLAN type system. However, by incorporating the user density information and the terrain-based channel attenuation we are able to estimate the interference levels one could expect in a practical system. Since WLAN type system is a strong candidate for TVWS usage [6], it is important to extend the work proposed here with more precise information about the impact of actual protocols and user demands.

The paper is organized as follow. In Section II we describe the system model and the modification proposed to the PPP model. In Section III we illustrate the performance of the proposed model by evaluating the interference in an example area in Finland. In Section IV we conclude the paper.

\section{System MODEL}

The spectrum utilization in the TVWS is allowed if the SINR requirement at the TV receivers is not violated. Since it is impossible to estimate the reception quality at each receiver, we follow the approach adopted by ECC [3] and describe the space by discrete points, pixels. The TV reception quality is calculated at the selected pixels in the TV coverage area. The 
interference at these test pixels is generated from the discrete pixels covering the area used by the secondary transmitters.

The area used in the numerical computations is illustrated in Fig. 1a and the corresponding user density in this area in Fig. 1b. We use the following notation: the number of test pixels in TV coverage area is $M$ and the secondary deployment area is discretized with $N_{p}$ pixels. For computing the slow fading parameters we surround each TV test pixels with $L$ nearby locations. The slow fading parameters from the $n$th interfering pixel to the $m$ th test pixel is evaluated based on the path loss values to these $m_{\ell}$ locations. The estimation of the slow fading parameters is described at the end of this section.

In numerical computations we use $M=10$ test pixels inside the TV coverage area. Each pixel is surrounded by $L=100$ test points. The deployment area of secondary transmitters is a rectangular which is covered by a square lattice. We call the lattice element as the coverage area of the pixel. Each pixel is described by the point in the center of its coverage area. In our computations we use $N_{p}=89 \times 154$ pixels.

At each TV test pixel we compute the SIR as

$$
\mathrm{SIR}=\frac{S}{I}
$$

where $S$ is the TV signal level and $I$ is the aggregate interference from the secondary transmitters.

The interference is computed as the sum over interfering powers

$$
I=\sum_{n} P_{n} g_{n m}
$$

where $P_{n}$ is the interference power emitted from the pixel $n$ and $g_{n m}$ is attenuation from the secondary pixel $n$ to the test pixel $m$.

The attenuation between the pixels $n$ and $m$ is computed by using terrain information and Longley-Rice channel model implementation in SPLAT! computer package [7].

With discrete pixels, the attenuation is not modeled from each secondary transmitter to each TV test pixel but from the secondary pixel $n$ to the test pixel $m$. The interference from the pixel $n$ is computed as the sum of the powers of the transmitters inside the pixel coverage area.

We model the WLAN type of secondary spectrum usage. We know the household density in the secondary deployment area and assume that each household has one secondary transmitter. Each transmitter has transmission power $P_{t}$ and activity factor $\nu$. The power emitted from the $n$th secondary pixel is $P_{n}=K \nu P_{t}$ where $K$ is the number of households inside the coverage area of pixel $n$.

We consider the slow fading by not only computing the attenuation, $g_{n m}$, from the pixel $n$ to test pixel $m$ but to each test point $\ell$ surrounding the test pixel $m, g_{n m_{\ell}}$. The interference distribution and SIR distribution are expressed as the histograms of the interference in all $m_{\ell}$ test points.

\section{A. PPP based interference modeling}

The Poisson point process (PPP) provides a simple approach that allows to model interference from randomly located

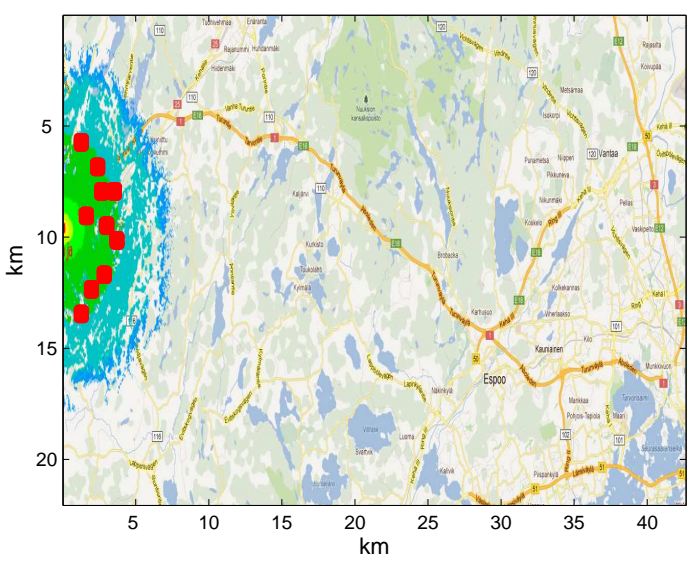

(a) Terrain and TV coverage area.

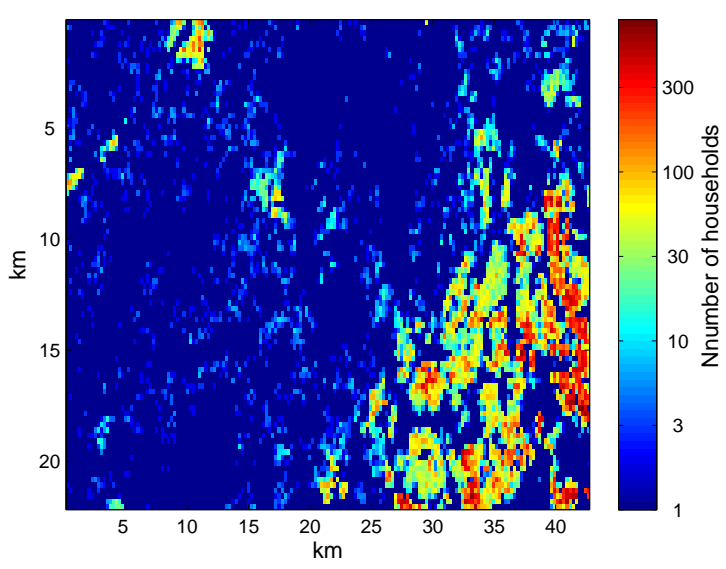

(b) Density of households.

Fig. 1: The system set up used in modeling.

non-synchronous transmitters. We can use such model for describing interference from a WLAN transmitter. In order to model the interference in the above described environment we modify the PPP model to incorporate the given user density and channel propagation information.

The interference from a single transmitter at the location $a$ is expressed as $I_{1, a}\left(P_{t}, x\right)=P_{t} g_{a i} x$ where $g_{a i}$ is the average path loss from the location $a$ to the TV test point $i$ and $x$ is a random variable describing the fading and having distribution $p_{X}(x)$. In our analysis the test point $i$ can be either a test pixel $m$ or a test point $m_{\ell}$. The distribution of one user located randomly into the area $A$ is

$$
I_{1}(x)=P_{t} x E\left\{g_{a i}\right\}=P_{t} x \int_{A} g_{a i} p_{A}(a) d a
$$

where $p_{A}(a)$ is the probability of finding the user at the location $a$.

For uniform user distribution $p_{A}(a)=\frac{1}{A}$. The moment 
generating function of the interference $I_{1}$ is

$$
M_{1}(s)=\int_{x} p_{X}(x) \int_{A} e^{s x P_{t} g_{a i}} \frac{1}{A} d a d x .
$$

For $k$ independent users the moment generating function can be expressed as

$$
E\{M(s) \mid k\}=M_{1}(s)^{k} .
$$

The PPP model expresses the moments of the interference from multiple users by averaging the moment distribution of the number of transmitters in the area $A$.

$$
F(s)=\sum_{k=0}^{\infty} E\{M(s) \mid k\} \operatorname{Pr}(k) .
$$

The probability of having $k$ independently located users in an area is given by the Poisson distribution

$$
\operatorname{Pr}(k)=N^{k} \frac{e^{-N}}{k !}
$$

where $N$ is the average number of users in the area $A$.

By using (7) and (5) in (6) we get the moment generating function for the aggregate interference distribution [8]

$$
F(s)=\sum_{k=0}^{\infty} E\{M(s) \mid k\} \frac{N^{k} e^{-N}}{k !}=e^{N\left(M_{1}(s)-1\right)} .
$$

The mean and the second moment of the aggregate interference can be computed from the first two derivatives of (8).

$$
\begin{aligned}
E\left\{I_{P}\right\} & =\left.F^{\prime}(s)\right|_{s=0}=\frac{N}{A} P_{t} E\{x\} \int_{A} g_{a i} d a \\
E\left\{I_{P}^{2}\right\} & =\left.F^{\prime \prime}(s)\right|_{s=0} \\
& =\frac{N}{A} P_{t}^{2} E\left\{x^{2}\right\} \int_{A} g_{a i}^{2} d a+E\left\{I_{P}\right\}^{2}
\end{aligned}
$$

where $I_{P}$ stands for the aggregate interference from the PPP.

In the following, we adapt the moments computed in (9) and (10) to describe the interference computed in (2). For that we modify these equations to incorporate variations of channel models and user demand.

1) PPP model for nonuniform users density: The moment generating function (8) is derived for uniform user location probability distribution. The uniform assumption simplifies the analysis. The derivation of corresponding moment generating function for other distributions is very cumbersome. However, we notice that the moments are computed by integrating over the service area $A$. We can split the area into subareas $A_{t}$ and sum the moments over these areas. Inside each area the density can be assumed to be constant (uniform). The first two moments of the aggregate interference are

$$
\begin{aligned}
E\left\{I_{P}\right\} & =\left.F^{\prime}(s)\right|_{s=0}=\sum_{t} \frac{N_{t}}{A_{t}} P_{t} E\left\{x_{t}\right\} \int_{A_{t}} g_{a i} d a(11) \\
E\left\{I_{P}^{2}\right\} & =\left.F^{\prime \prime}(s)\right|_{s=0} \\
& =\sum_{t} \frac{N_{t}}{A_{t}} P_{t}^{2} E\left\{x_{t}^{2}\right\} \int_{A_{t}} g_{a i}^{2} d a+E\left\{I_{P}\right\}^{2}
\end{aligned}
$$

where $N_{t}$ is the average number of users inside the area $A_{t}$ and $x_{t}$ is a random variable describing the fading.

In shadow fading the random variable $x_{t}$ is assumed to have a log-normal distribution with mean and standard deviation 0 and $\sigma_{t}$ respectively, both measured in $\mathrm{dB}$.

2) Modeling the nonuniform attenuation: We are interested to compare how well a PPP model describes the interference from the considered WLAN network. The PPP model is parameterized by the channel model and the user density. We extract those parameters for the network described in the area in Fig. 1.

The simplest model for WLAN interference estimation is to assume the uniform distribution of transmitters and the same path loss model for each location in the considered area (Fig. 1). Usually the PPP model is used with the simple power law based attenuation and slow fading. The distance-based pathloss is

$$
g_{a i}=c \cdot r^{-\alpha}
$$

where $c$ is the attenutation constant, $r$ is the distance and $\alpha$ is the path loss exponent.

We assume that the transmissions from all locations inside an area $A_{t}$ are described by the same propagation model. The channel model parameters, $c_{t}, \alpha_{t}$ and $\sigma_{t}$ for the area $A_{t}$ are found by fitting the model (13) to the attenuation parameters numerically computed by using the Longley-Rice model. In order to do that, we fix the value of the pathloss attenuation exponent $\alpha_{t}$ and compute the standard deviation $\sigma_{t}$ and attenuation constant $c_{t}$ from the first two moments of the aggregate interference distribution.

We allocate a single transmitter at each secondary pixel inside the area $A_{t}$ and match the first two moments of the aggregate interference computed by using the power law and the terrain-based channel model. It is assumed that the aggregate interference follows the log-normal distribution. This assumption has been justified by simulations in [9]. The first two moments of a log-normal distribution with mean equal to $0 \mathrm{~dB}$ are

$$
\begin{aligned}
& E\left\{x_{t}\right\}=e^{\frac{\sigma_{t}^{2}}{2 \xi^{2}}} \\
& E\left\{x_{t}^{2}\right\}=e^{\frac{2 \sigma_{t}^{2}}{\xi^{2}}}
\end{aligned}
$$

where $\xi=10 / \log (10)$ is a scaling constant.

In order to compute the mean interference level by using the terrain-based model we compute the average interference value over the $L$ test points. By equating it to the mean interference level computed from the power-law model we get

$$
c_{t} \cdot e^{\frac{\sigma_{t}^{2}}{2 \xi^{2}}} \sum_{n \in A_{t}} r_{n m}^{-\alpha}=\frac{1}{L} \sum_{n} \sum_{\ell} g_{n m_{\ell}}
$$

where $r_{n m}$ is the distance from the $n$th secondary pixel inside the area $A_{t}$ to the $m$ th $\mathrm{TV}$ test pixel and the transmission power level has been cancelled out from both sides.

The second moment of the aggregate interference by using the terrain-based model should be computed over all pairs, $n_{1}, n_{2}$, of secondary pixels inside the area $A_{t}$. By equating the 
second moments obtained from power law based attenuation and terrain-based channel model we get

$$
\begin{aligned}
& c_{t}^{2} \cdot e^{\frac{\sigma_{t}^{2}}{\xi^{2}}} \cdot\left(e^{\frac{\sigma_{t}^{2}}{\xi^{2}}}-1\right) \cdot \sum_{n} r_{n m}^{-2 \alpha}= \\
& \frac{1}{L} \sum_{\ell} \sum_{n_{1}} \sum_{n_{2}} g_{n_{1} m_{\ell}} g_{n_{2} m_{\ell}}-\left(\frac{1}{L} \sum_{n} \sum_{\ell} g_{n m_{\ell}}\right)^{2} .
\end{aligned}
$$

By taking the square of (16) and dividing by sides with (17) we compute the variance of the slow fading for the area $A_{t}$

$$
\sigma_{t}^{2}=\xi^{2} \log \left(1+\frac{\left(\sum_{n} r_{n m}^{-\alpha}\right)^{2}}{\sum_{n} r_{n m}^{-2 \alpha}}\left(L \frac{\sum_{\ell} \sum_{n_{1}} \sum_{n_{2}} g_{n_{1} m_{\ell}} g_{n_{2} m_{\ell}}}{\left(\sum_{\ell} \sum_{n} g_{n m_{\ell}}\right)^{2}}-1\right)\right)
$$

The attenuation constant $c_{t}$ is derived after replacing (18) into (16)

$$
c_{t}=e^{-\frac{\sigma_{t}^{2}}{2 \xi^{2}}} \cdot \frac{\sum_{n} \sum_{\ell} g_{n m_{\ell}}}{L \cdot \sum_{n} r_{n m}^{-\alpha}}
$$

With the parameters $\alpha_{t}, c_{t}$ and $\sigma_{t}$ at hand, the first two moments of the aggregate interference can be computed. The integration in (11) can be approximated as a summation over the secondary pixels inside the area $A_{t}$

$$
\int_{A_{t}} g_{a i} d a \approx A_{p} \cdot c_{t} \cdot \sum_{n \in A_{t}} r_{n m}^{-\alpha}
$$

where $A_{p}$ is the pixel coverage area size and the term $A_{p} \cdot N_{t} / A_{t}$ is equal to the average number of transmitters per secondary pixel in the area $A_{t}$.

Similarly, for the computation of the second moment (12) the following approximation is used

$$
\int_{A_{t}} g_{a i}^{2} d a \approx A_{p} \cdot c_{t}^{2} \cdot \sum_{n \in A_{t}} r_{n m}^{-2 \alpha} .
$$

\section{NUMERICAL EXAMPLES}

We illustrate the interference from the WLAN network located in Helsinki area. The selected area is illustrated in Fig. 1a and the household density in this area is illustrated in Fig. 1b. The total number of households in the considered area is approximately 200000 . The considered secondary system covers area of $22 \times 42 \mathrm{~km}^{2}$ and the household density is approximately 200 households per $\mathrm{km}^{2}$.

The TV coverage area is visible in Fig. 1a. We select $M=$ 10 test pixels in the TV coverage area. Each test pixels is surrounded by $L=10 \times 10$ test points $\left(m_{\ell}\right)$. The test points are located in the grid with center at the test pixel $m$ and distance between the grid points is $50 \mathrm{~m}$.

The secondary system area is covered by $N_{p}=89 \times 154$ pixels. The pixel coverage area is equal to $250 \times 250 \mathrm{~m}^{2}$.

We compute the attenuation $g_{n m_{\ell}}$ between secondary test pixel $n$ and test points $m_{\ell}$ by using Logley-Rice channel model implementation in SPLAT! software package [7] and terrain information from digital elevation terrain data [10].

By using our parameter settings, the smallest area the PPP model can have is one pixel, $250 \times 250 \mathrm{~m}^{2}$. In Fig. 2 we

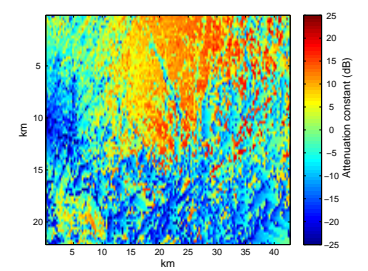

(a) $10 \log _{10}\left(c_{n}\right)$

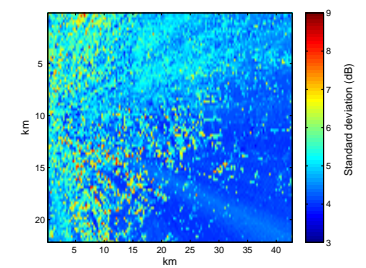

(c) $\sigma_{n}$

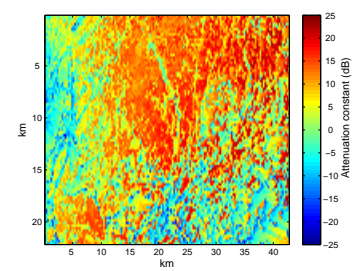

(b) $10 \log _{10}\left(c_{n}\right)$

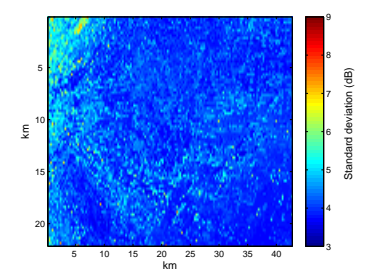

(d) $\sigma_{n}$
Fig. 2: Pathloss attenuation constant and standard deviation, both expressed in $\mathrm{dB}$, from each secondary pixel $n \in A$ towards two different TV test pixels.

illustrate the path loss model match between the Longley-Rice and the power law model (13). In the matching we selected the path loss exponent to be $\alpha=3.5$. In Fig. 2c and Fig. 2d the standard deviation ranges from $3 \mathrm{~dB}$ to $9 \mathrm{~dB}$ and similar values are observed for other test points too. One observes that transmissions from neighboring pixels are in general described by similar attenuation constants and slow fading standard deviations. In addition, the propagation characteristics to different TV test pixels are in general different.

We compute the interference for the WLAN type network where each household is assumed to have one transmitter and the transmission activity is $\nu=0.05$. The transmission power for each secondary transmitter is taken equal to $P_{t}=10$ $\mathrm{mW}$. In addition, WLAN transmitters located at a distance smaller than $400 \mathrm{~m}$ to the TV coverage area are not allowed to transmit. The power emitted from each secondary pixel is the number of households in the pixel area times the transmission power level $10 \mathrm{~mW}$ times the activity factor $\nu$. In PPP model the average amount of users $N_{t}$ in the area $A_{t}$ is computed by scaling the total number of households in the area with the activity factor.

In Fig. 3 we illustrate the cumulative distribution function (CDF) of the computed interference with different area sizes. In that figure the continuous blue line describes the interference computed from (2). We see that the PPP model overestimates the interference level. Compared to the exact distribution, the difference in the mean interference levels is about $8 \mathrm{~dB}$. In our system set up most of the households are concentrated far from the TV protection contour while, the PPP assumes them to be uniformly distributed inside the area. However, already for 25 areas, splitting the considered area to $5 \times 5$ areas, the PPP based estimation is relatively close to the actual interference level.

The approximation accuracy in the high values of the 


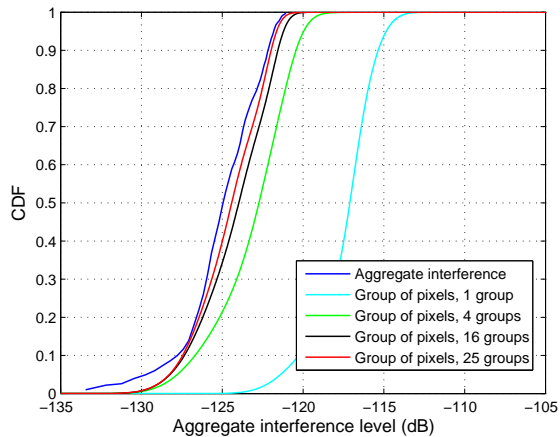

Fig. 3: Distribution of the aggregate interference at the TV test points.

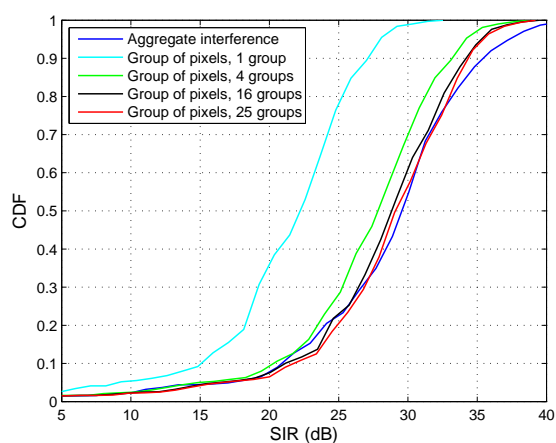

Fig. 4: SIR distribution at the TV test points.

interference is particularly of interest as it determines whether the TV protection criteria are violated or not. In the high end, the approximation error becomes negligible. Good match at the upper tail of the interference distribution means that the PPP model describes well the lower tail of SIR distribution. This is illustrated in Fig. 4. In the SIR computation the TV signal power is computed based on the transmission power of the given TV transmitter and the Longley-Rice model. One can see that for our parameter settings a $20 \mathrm{~dB}$ SIR target is satisfied with outage probability $10 \%$. These values are considered to not violate the protection criteria of the TV receivers.

\section{CONCLUSION}

In this paper we compute the aggregate interference from a WLAN type secondary network located in the TVWS. As an illustrative case study we use Helsinki area in Finland. In the computations we use the terrain and user density information of the selected area.

In the illustrative case we computed path loss values by the Longley-Rice model. We show how these relatively precise channel attenuation values can be approximated with simple power law and shadow fading based channel model. The proposed approximation approach can be applied also, when the attenuation values are not acquired from the computations but, for instance, from the measurements.

We describe how the well known PPP model has to be modified in order to describe the aggregate interference in selected realistic environment. The modified PPP model provides surprisingly good match to the actual interference level. However, the good description is acquired only after careful selection of the model parameter values. For instance, if to assume a simple uniform demand in the whole secondary system coverage area the interference levels provided by the PPP model are far from the actual observed values.

The treatment in this paper is an initial step in modeling the WLAN system interference. It is well known that a simple PPP process does not describe the impact of WLAN systems MAC protocols. How to incorporate such details into the interference model is the subject of the future research.

\section{ACKNOWLEDGMENT}

The authors would like to thank the European Union for providing partial funding of this work through the EU FP7 project INFSO-ICT-248303 QUASAR.

\section{REFERENCES}

[1] P.C. Pinto, M.Z. Win, "Communication in a Poisson Field of Interferers Part I: Interference Distribution and Error Probability," IEEE Trans. Wir. Commun., vol. 9, no. 7, pp. 2176-2186, Jul. 2010.

[2] J. Kingman, "Poisson Processes," Oxford University Press, 1993.

[3] ECC, Report 159 (under public consultation): Technical and operational requirements for the possible operation of cognitive radio systems in the white spaces of the frequency band 470-790 MHz, Sept. 2010.

[4] G.A. Hufford, A.G. Longley, and W.A. Kissick. "A guide to the use of the Irregular Terrain Model in the area prediction mode," Technical Report 82-100, NTIA, 1982

[5] K. Gulati, B.L. Evans, J.G. Andrews and K.R. Tinsley, "Statistics of CoChannel Interference in a Field of Poisson and Poisson-Poisson Clustered Interferers," IEEE Trans. Sig. Process., vol. 58, no. 12, Dec. 2010.

[6] J. Kronander, M. Nekovee, K.W. Sung, J. Zander, S.L. Kim, and A. Achtzehn, "QUASAR scenarios for white space assessments and exploitation", in Proc. Conf. URSI EMC, Sept. 2010.

[7] RF Signal Propagation, Loss, And Terrain analysis tool for the spectrum between $20 \mathrm{MHz}$ and $20 \mathrm{GHz}$. Available at http://www.qsl.net/kd2bd/ splat.html.

[8] A. Papoulis, "Probability, random variables and stochastic processes," 3rd edition, MCGraw-Hill, International editions, 1991.

[9] K. Ruttik, K. Koufos and R. Jäntti, "Computation of aggregate interference from multiple secondary transmitters," IEEE Commun. Lett., vol 15, no. 4, pp. 237-439, Apr. 2011.

[10] Digital elevation terrain data for North Eurasia, Available at: http://www. viewfinderpanoramas.org/dem $3 . \mathrm{html}$. 